\title{
Editorial
}

\section{Do Not Be Afraid of the Robot}

\section{No le tenga miedo al robot}

\author{
Camilo Giedelman ${ }^{1}$ Rafael Clavijo ${ }^{1}$ Hernán Aponte ${ }^{1}$ \\ ${ }^{1}$ Minimally Invasive Surgery Group, Laparoscopy and Robotics, Clínica \\ de Marly, Hospital de San José, Bogotá, Colombia \\ Urol Colomb 2019;28:97-99.
}

Minimally invasive surgery is one of the areas of greatest development in surgical specialties in several parts of the world, and Colombia has not been the exception. In urology, as in all specialties, the evolution in technology has optimized the diagnosis and the treatment of most of the pathologies. The purpose has always been that the patients who undergo surgery have a pleasant, less stressful experience, without compromising the perioperative and postoperative results.

In this search, 9 years ago, the Clínica de Marly was the first site to bring the Da Vinci (Minimally Invasive Surgery Group, Laparoscopy and Robotics, Urologic Team, Clínica de Marly, Hospital de San José, Fundación Universitaria de Ciencias de la Salud, Bogotá, Colombia) robot. Since then, both in our institution and in others located in Bogotá, which later acquired this same technology, a large number of surgical interventions have been successfully carried out. The great motivation for this is supported by the fact that the robotic approach allows surgeons to increase the level of precision of their interventions, and as the experience of the operative team increases, procedures are performed with greater safety and fewer complications, with successful oncological and functional results comparable with what is achieved with techniques such as open or laparoscopic, always clarifying that any operative technique should be performed by expert surgeons.

Robotic surgery allows surgeons to increase the level of precision of their interventions, achieving, with a lower learning curve, the same oncological and functional results achieved by open and laparoscopic surgeons who handle large surgical volumes. However, the human factor is still essential in obtaining good results in robotic surgery: good robotic surgeons with more experience will probably have better results.

We must consider that open surgery has already reached its maximum development. In its beginnings, robotic surgery in urology has achieved the same results as open surgery, which makes us wonder how far can robotic surgery go. However, in robotic surgery, there is constant development that will take this technology to unsuspected levels. Examples of this are magnified vision, high definition, the possibility of practicing microscopic approaches, being able to see beyond what is seen (indocyanine green immunofluorescence), image fusioning during surgery, development of tactile sensations, equipment miniaturization, and new software, among others, are some of the expected developments.

However, the current robot has clear disadvantages that must be handled so they can be corrected in the future. One of them is the bulky size of the equipment, requiring large operating rooms. In addition, the robot requires a large number of delicate connections inside the surgery room that can cause accidents.

The main disadvantage at present is undoubtedly the high costs of the equipment and its operation fee; this is due to the fact of the monopoly that exists at the moment by the manufacturing company, which has caused many groups to question the cost-efficiency of the treatments. With the arrival of new robots in the market, these costs will probably decrease and surgery will become more affordable and cost-effective.

\section{The Recent Alert of the Food and Drug Administration}

On February 28, an alert that involved robotic surgery on the website of the Food and Drug Administration (FDA), which is the entity in charge of monitoring and controlling medicines and medical devices in the United States, was published; the statement warns about the use of robot-assisted surgical devices for surgeries related to cervix and breast cancer. The FDA bases its recommendation on two investigations published last year in the New England Journal of Medicine. Both studies question the use of minimally invasive surgery specifically in cases of women with cervical cancer, arguing that patients who underwent surgery with this technique had a greater possibility of relapse and lower total survival compared with those operated with open surgery.

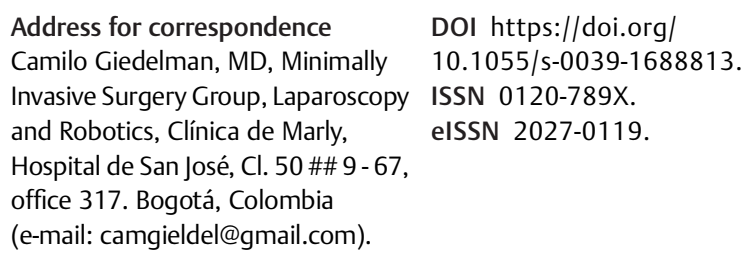

Copyright @ 2019, Sociedad Colombiana
de Urolonse terms
Revinter Publicações Ltda., Rio de Janeiro, Brazil. Todos los derechos reservados. 
This information was replicated by several media, and this generated a great impact both in patients and in the medical community, and although some points of the statement are true, in others it is convenient to have greater clarity due to the fact that we consider that there are some inaccuracies in the interpretation of the FDA communication.

The study on which the alert is based is the Laparoscopic Approaches to Cervical Cancer (LACC); it is a well-designed prospective randomized study, and that is why Gynecology Oncology groups are on alert throughout the world. Now, there are some observations that can be made to the study. One is that the group of procedures performed by MI (Minimally invasive surgery) included a small number of patients assisted by robot, only $15 \%$, while $85 \%$ were performed by laparoscopy, and there may be too few to establish statistically significant results against the robot. There is no doubt that the alert should be carefully considered, but more studies are needed to understand the cause of the findings and thus be clearer when relating them to the operative technique.

Ideally, subsequent studies should individualize the management and the follow-up for each type of cancer, since several surgical specialties have had very good results in procedures performed by the robotic approach. To date, there are $>18,000$ scientific articles on robotic surgery, and many of these publications are comparisons of the techniques in which it is not possible to demonstrate the inferiority of any of the approaches in comparison with the other. It has even become clear that there may be superiority favoring the robotic technique in the immediate postoperative and postoperative period (less bleeding, less pain, shorter hospitalization time, and shorter time to return to baseline conditions).

Surely, with the alert, the gynecological procedures for cancer treatment are going to have an evaluation, and the scientific societies will be responsible for making the respective adjustments in some processes defining what should be done and which procedures should be temporarily or permanently restricted.

The path of robotic surgery will continue, and with situations like those commented on the alert, the route will be perfected. The conviction lies not only in a great technological team, but also in the preparation and in the experience that surgeons have acquired for $>20$ years since the robot first arrived at the operating rooms.

\section{Radical Prostatectomy: A Successful Model of the Robotic Technique}

One of the techniques in urology that has evolved the most from the arrival of the robot is radical prostatectomy. And there were 2 main reasons for this. In the first place, the diagnosis of prostate cancer is susceptible to early detection, and when caught in the early stages, it is clear that surgeries can cure the disease, but we all know that it is a high technical demand procedure for any of the approaches; and this is where robotic surgery can take advantage, since the work area is better addressed and a better surgical exposure can be achieved, as well as greater ranges of movement.
In $<2$ decades, radical robotic prostatectomy became the most used surgical technique for the treatment of prostate cancer in the world. Today, $>85 \%$ of the radical prostatectomies are performed by this approach in the US, a place where critical analysis is done under rigorous scientific scrutiny and not by expert opinions, as it occurs in countries like ours.

Regarding the main objective of the procedure, which is cancer control, robotic prostatectomy responded to people who looked at robotic prostatectomy with skepticism and showed that biochemical recurrence-free survival is comparable to open prostatectomy in a long-term follow-up. Perioperative results are other important aspects to evaluate, in which the robot gave better results compared with open surgery. There was a significant decrease in estimated blood loss, complication rates $(6.6 \%$ for robotic versus $10.3 \%$ for open), and length of hospital stay.

By using potential, validated quality of life questionnaires, it was found that patients undergoing PRAR (Roboticassisted radical prostatectomy) had higher scores and faster return to their baseline functions compared with patients who underwent open prostatectomy. The results obtained so far for sexual function and continence were similar when both groups were compared.

Nowadays, surgical technologies are under continuous critical evaluation, but despite this, robotics has prevailed. Negative criticisms are less frequent and, with this, several skeptical urological groups have changed their direction when adopting this surgical technology.

\section{Note}

- Up to March 2019, we have performed 200 cases of radical prostatectomy, with an average hospitalization time of 2.2 days, low rates of peri- and postoperative complications $(<3 \%)$; only 1 patient converted to open surgery; rates of disease relapse, return to continence, and return of sexual function were comparable to the statistics of high-volume centers of the world. As more cases are conducted, the results are better, as expected, and this has brought greater satisfaction to the patients and to the group of surgeons. We believe that the key to the success of the program has been the training carried out previously, mixed with the experience acquired over time; this translates into safety and good results.

\section{Discussion and Conclusions}

The basic principles of surgery are still applied in robotics: experience counts and prolonged practice provides knowledge and skills. In this manner, the potential benefits provided by the technology will be better exploited and this will be reflected in better outcomes for the patients. The main advance is to find better techniques to improve the functional results and raise the evaluation standards.

However, it is important to emphasize that the results obtained by robotic surgery do not depend so much on the technique as on the experience of the surgeon, that is why it is important that patients, before making the choice for the 
surgical approach, understand that the planning for any procedure is a decision that must be shared between the surgeon and the patient, discussing all the risks, the benefits, and the alternatives of each procedure. A surgeon should inform his patient how many cases he has attended, the risk of complications, and how many times they have been presented in his experience, as well as the results obtained in the long-term. At this point, we want to be emphatic that the numbers that the surgeon should give the patient should not be from book statistics, they should be told by the personal experience data, otherwise this could generate false expectations to the patients, and that could be a deception that the patient should not assume. It is necessary for the person to be aware that not only is he/she undergoing a certain operative technique, but that he/she is putting his/ her life in the hands of a surgeon who will offer him/her the best alternative, and that is precisely what the FDA says within its surgical recommendations.

In conclusion, we must talk about what we have done in our institution, because we are an example of what can be achieved. The short- and mid-term results of robotic prostatectomy have shown oncological and functional results that are, at least, comparable with the reports of expert surgeons (to be published soon), with similar oncological and functional results, as well as with the potential benefits of less bleeding and shorter convalescence that support and justify the widespread adoption and application of this procedure.

We clearly recognize the importance of the analysis that is still being carried out in the world about the technique, and there must be a long-term follow-up to continue critically evaluating and defining the role of robotics in the treatment of prostate cancer and other urological pathologies.

The robotic surgery history is still being written; it is the first part of a digital era that the surgical world recently entered after having exploited to the maximum the open and laparoscopic techniques. It is true that measures adopted in the last 3 decades have been important, but there is still a long way to go. You should not be afraid of the robot. On the contrary, we should take advantage of what technology offers us, always with a critical attitude but with enthusiasm, not letting the opportunity of progress that has reached the surgical scenario pass away.

Conflicts of Interests

The authors have no conflicts of interests to declare.

\section{Related readings}

1 Giedelman C, Abdul-Muhsin H. El impacto de la cirugía robótica en Urología. Actas Urol Esp. 2013;37(10):652-657

2 US Food and Drug Administration Home Page, Caution When Using Robotically-Assisted Surgical Devices in Women's Health including Mastectomy and Other Cancer-Related Surgeries: FDA Safety Communication, February 28, 2019, https://www.fda.gov

3 Ramirez P, Frumovitz M. Minimally Invasive versus Abdominal Radical Hysterectomy for Cervical Cancer. N Engl J Med 2018; 379:1895-1904. November 15, 2018

4 Rassweiler J, Autorino R. Future of robotic surgery in urology. BJU Int 2017;120:822-841 